\title{
Fractal structure of the Horsehead nebula (B 33)
}

\author{
S. Datta \\ Department of Applied Mathematics, University of Calcutta, 92 APC Road, Calcutta 700 009, India \\ Received 6 December 2001 / Accepted 5 September 2002

\begin{abstract}
Analysis of the CCD image of the Horsehead nebula (B 33), taken in the $\mathrm{H} \alpha(6561 \AA)$ using the $2.34 \mathrm{~m}$ Vainu Bappu Telescope (VBT) at Kavalur, India, is performed to test its fractal structure. Ten sample readings of the box dimension of this image were taken using a fractal analysis software, giving an average value of 1.6965725. The sample dimensions were found to be different from the topological dimension of one. Importantly, the box dimension of B 33 was not found to be significantly different from that of the Julia set (box dimension 1.679594) with $c=-0.745429+0.113008 i$. This provides compelling evidence to show that the structure of the Horsehead nebula is not only fractal, but also that its geometry can be described by the Julia function $f(z)=z^{2}+c$, where both $z$ and $c$ are complex numbers.
\end{abstract}

Key words. ISM: clouds - ISM: general

\section{Introduction}

The Horsehead nebula (B 33) located in the northern Giant Molecular Cloud (GMC), Orion B, of the Orion cloud complex, is arguably one of the most spectacular nebulae. It is about 2 square degrees in size at a distance of $450 \mathrm{kpc}$ (Malin et al. 1987), centered at RA 5h 40m 59.0s; Dec 5 27'29.99'(J2000). It is found to be connected to its parental cloud (Orion B) and is a young evolving cloud with a virial mass of $35 M_{\odot}$ and radius $0.17 \mathrm{pc}$ (Lada et al. 1991) with average density $3 \times 10^{4} \mathrm{~cm}^{3}$. It is exposed to the destructive influence of the Trapezium , which lies $30^{\prime}$ to the east and $\zeta$ Ori which lies $30^{\prime}$ to the north (Kramer et al. 1996). The recombination region is IC 434. Kramer et al. (1996) have reported that Herbig Haro (HH) objects, IR point sources, condensations in $\mathrm{NH}_{3}$ and ${ }^{13} \mathrm{CO}$ are found within B 33 and that ${ }^{13} \mathrm{CO}$ maps show the existence of a clump in the central part of the Horsehead of virial mass $95.4 M_{\odot}$, radius $0.22 \mathrm{pc}$, density $2 \times 10^{3} \mathrm{~cm}^{-3}$, temperature $5.8 \mathrm{~K}$ and velocity $10.5 \mathrm{~km} \mathrm{~s}^{-1}$. Spectra also show that there is ongoing star formation inside B 33.

The structure of molecular clouds have been observed to follow a power-law relation (Larson 1981; Elmegreen 1999; Williams 1997). Attempts to determine this relation using various methods (Hetem \& Lepine 1993; Stutzki et al. 1998; Kramer et al. 1998) have been made. Kramer et al. (1998) carried out an analysis on the images of the Orion B region, amongst others, using automated software and found that the clump mass spectra was consistent with a power-law,

$\mathrm{d} N / \mathrm{d} M \propto M^{-\beta}$

with $\beta=1.72 \pm 0.09, M$ being the mass of the clump and $N$ the number of clumps with mass $\mathrm{M}$. In this paper, the author

^ e-mail: srabani@cucc.ernet.in analyzes the structure of B 33 in order to determine its geometry and estimate its dimension. The details of the observations carried out using the $2.34 \mathrm{~m} \mathrm{VBT,} \mathrm{Kavalur,} \mathrm{are} \mathrm{given} \mathrm{in} \mathrm{Sect.} \mathrm{3,}$ the software and statistical analysis techniques used are given in Sect. 4 and the results and implications for cloud formation theory is discussed in Sect. 5.

\section{Mathematical background}

Fractals are self-similar objects with fine structure (Mandelbrot 1982; Falconer 1997; Peitgen et al. 1992). Examples of fractal objects include the von Koch curve, the Sierpinski gasket, the Cantor set and the Julia set given by the equation

$f(z)=z^{2}+c$

where $z$ and $c$ are both complex quantities. Quantification of fractal objects is achieved by measuring their dimensions. To do this, the fractal object is considered as a non-empty compact set of a metric space (Falconer 1997). An arbitrarily small number, $\epsilon$, is chosen and the number of open balls, $N(\epsilon)$, of radius $\epsilon$ covering the set is counted. Then, the Kolmogorov dimension (also known as Minkowski dimension) of the set is defined as

$K \operatorname{dim} K=\lim _{\epsilon \rightarrow 0} \sup \frac{\log N(\epsilon)}{\log (1 / \epsilon)}$.

Since such a method is difficult for practical measurements, an alternative dimension, called the grid dimension or box counting dimension, is used. In this method, a grid is placed over the object of mesh size $\epsilon$, and the number of grid boxes that contain part of the object, $N_{\mathrm{g}}(\epsilon)$, is counted. The grid dimension is defined as

$\operatorname{griddim} K=\lim _{\epsilon \rightarrow 0} \frac{\log N_{\mathrm{g}}(\epsilon)}{\log (1 / \epsilon)}$. 
The grid dimension is equivalent to the Kolmogorov dimension if the set is non-empty.

The geometrical shape of the Horsehead can be explained by considering the gas as a fluid. The equations of fluid motion used are the equation of continuity in the general case, the equation of continuity in the incompressible case and the Navier-Stokes equation, respectively, given by

$$
\begin{aligned}
& \frac{\delta \rho}{\delta t}+\nabla \cdot \boldsymbol{v}=0 \\
& \nabla \cdot \boldsymbol{v}=0 \\
& \frac{\delta \boldsymbol{v}}{\delta t}+\boldsymbol{v} \cdot \nabla \boldsymbol{v}=F-\frac{1}{\rho} \nabla p+\frac{\mu}{\rho} \nabla^{2} \boldsymbol{v}+\frac{1}{\rho}\left[K+\frac{1}{3} \mu\right] \nabla \cdot \boldsymbol{v}
\end{aligned}
$$

where $\rho$ is the density of the fluid, $\boldsymbol{v}$ is the fluid velocity at a point inside it at any particular time $t, F$ is the total surface forces on the fluid, $\mathrm{p}$ is the fluid pressure at that point, $\mu$ is the viscosity of the fluid and $K$ is a constant (Paterson 1992).

Since the Navier-Stokes equation is non-linear in $v$, the motion is chaotic and so we apply a non-linear iterative map from $v_{t} \mapsto v_{t+1}$ given by (Datta 2001b)

$v_{t+1}-v_{t}=v_{t}^{2}-v_{t}+c$.

This map is analogous to the Navier-Stokes equation in the incompressible case with the following representation:

$$
\begin{aligned}
& v_{t+1}-v_{t} \equiv \frac{\delta v}{\delta t} \\
& v_{t}^{2} \equiv-\boldsymbol{v} \cdot \nabla \boldsymbol{v} \\
& v_{t} \equiv v \nabla^{2} \boldsymbol{v} \\
& c \equiv F-\rho^{-1} \nabla p .
\end{aligned}
$$

The set of iterates $v_{t}$ starting from an initial value $\omega$ at time $t=0$, the orbit of the map (8), gives rise to a fractal with an attractor and a basin of attraction and so may be called a dissipative dynamical system (Frisch 1999).

\section{Observations}

The image of the Horsehead nebula (RA 5h 49m 58.6s; Dec $2^{\circ} 27^{\prime} 23.84^{\prime \prime}$ ) in the $\mathrm{H}_{\alpha}$ at $6563 \mathrm{~A}$ (bandwidth $50 \AA$ ) was observed with the $2.34 \mathrm{~m} \mathrm{VBT}$ at the Vainu Bappu Observatory (VBO) in Kavalur, India on 2nd April 2000. The exposure time was $30 \mathrm{~min}$ (Fig. 1). The VBT is a Cassegrain with a focal ratio of $f / 3$ at the prime focus. The data were acquired on a Photometrics CCD camera. The Tektronix CCD chip with $1024 \times 1024$ pixels was placed at the prime focus of the VBT where the plate scale is $0.66^{\prime \prime}$ per pixel, giving a field of view of 11.26 square arc min of the sky. The gain of the CCD is 8.9 electrons and the read-out-noise is 10 electrons. The image was bias subtracted and flat-fielded and cleaned of cosmic rays using IRAF Image Analysis software (NOAO, Arizona, USA). Dark subtraction was found to be unnecessary since the camera was cooled with liquid nitrogen to a temperature of $-90{ }^{\circ} \mathrm{C}$. The seeing was found to be 4 arcsec. Guide stars were found from the Hubble Space Telescope (HST) Guide Star Catalogue.

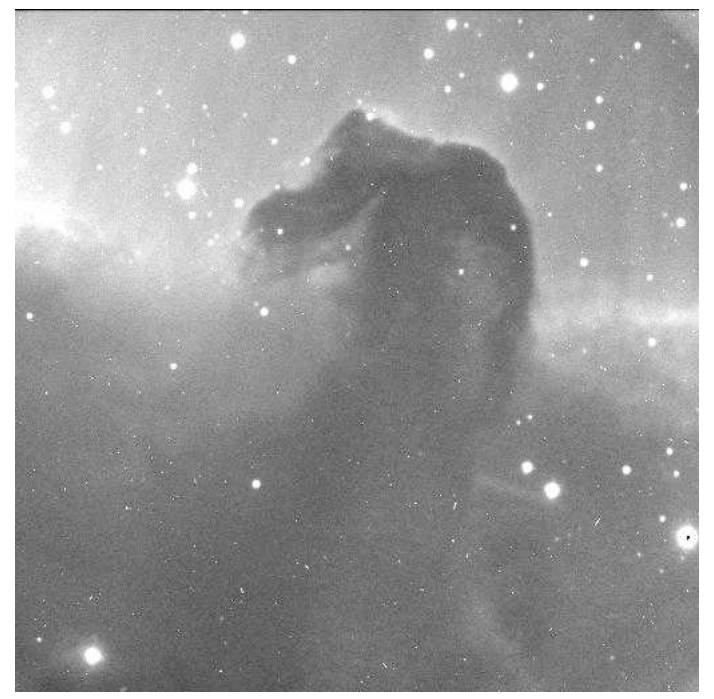

Fig. 1. $\mathrm{H}_{\alpha}$ image of the Horsehead nebula (B 33) taken from the $2.34 \mathrm{~m}$ VBT on 2nd April 2000.

\section{Method}

The fractal (box) dimension of B 33 was estimated using an automated dimension analysis software, Benoit 1.3 , procured from Trusoft International Inc., St. Petersburg, USA. Benoit has been reviewed (Seffens 1999) and its performance has been found to be satisfactory. For the analysis, a grid is overlayed on the image and a box is counted if there is a dark cloud in it as well as an edge, where an edge is a boundary between the dark cloud and the HII region. A set of 10 measurements were taken at fixed grid rotation increments of 15 degrees. An average dimension was calculated and the number of iterations giving the slope with the least standard deviation (SD) while being nearest to the average was chosen. The percentage error in estimating the slope for known fractals with Benoit 1.3 varies from 0.47 (Sierpinski gasket) to about 2 (Koch curve). Log-log graphs were then plotted (Fig. 2) of the reciprocal of the side length of the square against the number of outline-containing squares. Ten further sets of measurements were taken with this selected number of iterations and box size decrease factors (Table 1). Normality of the sample populations was tested with the Shapiro-Wilkes test (Pearson \& Hartley 1972). The 1 percent significance limit for $\mathrm{W}$ for 10 samples is 0.781 . The calculated value of $\mathrm{W}$ is 0.01065 and so the population is normal. The Student's $t$ test of significance (Kapur \& Saxena 1982) was then applied to test for difference of the sample average from a mean value of unity (topological dimension) and also from the average dimension of the Julia set (Fig. 3) found by the same method. The calculated $t$ value is 16.52918 , while the 1 percent significance limit for the Student's $t$ test is 3.25.

\section{Conclusions}

Comparison of the topological dimension value of one with the average box dimension of B $33(1.6965725)$ at a 1 percent level of significance shows that the Horsehead nebula shape is a true fractal. Results also show that the box dimension of the Horsehead nebula is not significantly different from that of 


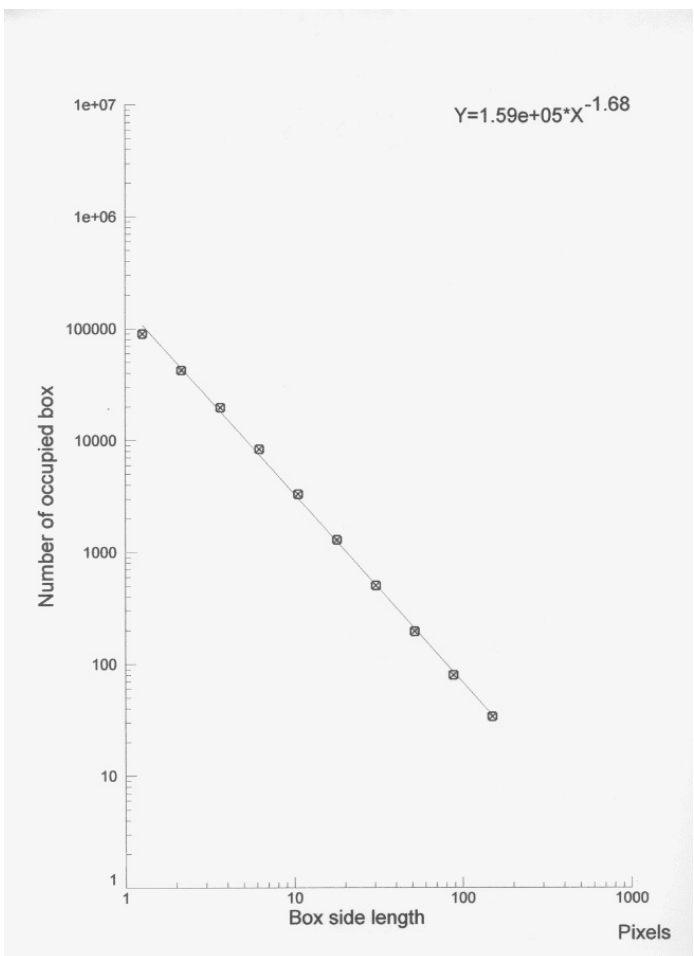

Fig. 2. Log-log plot of the reciprocal of side lengths of the square against the number of outline-containing squares. The slope gives the dimension.

Table 1. Box dimension of 10 readings measured on the $\mathrm{H}_{\alpha}$ image of B33 (Fig. 1) with Benoit 1.3. The grid rotation has been kept constant at 15 degrees. The average of 10 measurements is 1.696572 .

\begin{tabular}{ccccc}
\hline \hline $\begin{array}{c}\text { box } \\
\text { dimension }\end{array}$ & SD & $\begin{array}{c}\text { no. of } \\
\text { boxes }\end{array}$ & $\begin{array}{c}\text { Co-efficient } \\
\text { of box } \\
\text { decrease }\end{array}$ & $\begin{array}{c}\text { size of } \\
\text { largest } \\
\text { box }\end{array}$ \\
\hline 1.675196 & 0.016569 & 10 & 1.5 & 100 \\
1.71113 & 0.007594 & 9 & 1.5 & 80 \\
1.67231 & 0.008941 & 7 & 1.5 & 80 \\
1.73453 & 0.006216 & 9 & 1.4 & 50 \\
1.79839 & 0.001004 & 9 & 1.3 & 50 \\
1.68676 & 0.009777 & 9 & 1.7 & 40 \\
1.65873 & 0.008258 & 8 & 1.8 & 40 \\
1.68559 & 0.010693 & 10 & 1.5 & 40 \\
1.67440 & 0.010112 & 8 & 1.6 & 20 \\
1.66869 & 0.008465 & 7 & 1.7 & 10 \\
\hline
\end{tabular}

Table 2. Summary of box dimensions and test values of the figures measured.

\begin{tabular}{cccc}
\hline \hline Name & box dimension & $\begin{array}{c}\text { Shapiro-Wilkes } \\
\text { test value }\end{array}$ & $\begin{array}{c}\text { Student's } t \\
\text { value }\end{array}$ \\
\hline Julia set & 1.679594 & - & - \\
B33 & 1.696572 & 0.01065 & 16.52918 \\
IC434(col I) & 1.811718 & 0.00513 & 17.11578 \\
\hline
\end{tabular}

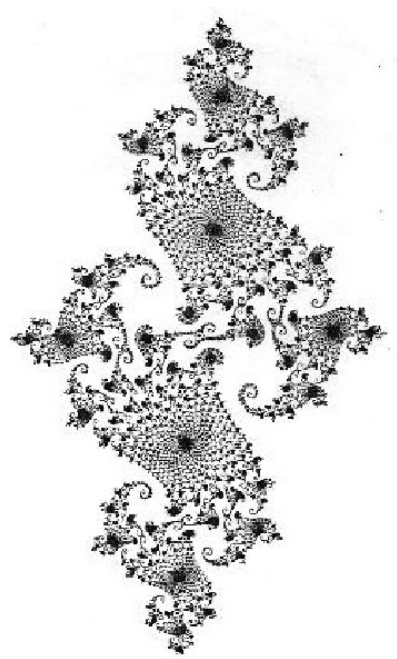

Fig. 3. The Julia set with $c=-0.745429+0.113008 i$ (Peitgen 1992). Box dimension is 1.679594 .

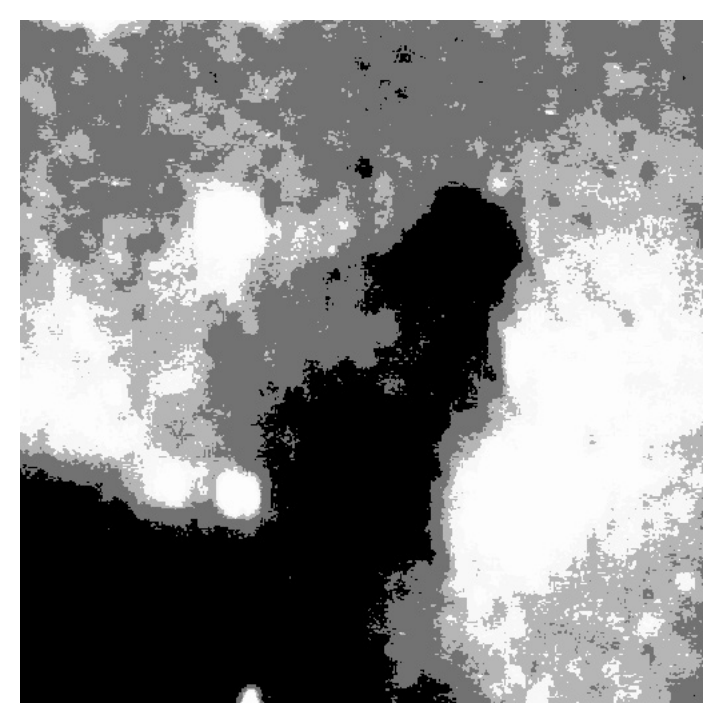

Fig. 4. DSS image of col. I in IC 434 (RA 5h 41m 48.89s; Dec $\left.-2^{\circ} 12^{\prime} 40.6^{\prime \prime}\right)$. Box dimension is 1.811718 .

the Julia set. Taking into consideration that there is a connection between the power-law index of the cloud mass distribution relation, Eq. (1), and the fractal dimension (Stutzki et al. 1998; Elmegreen \& Falgarone 1996), it is significant that its observed dimension is within the error limits $(\alpha=1.72 \pm 0.05)$ of Kramer et al. (1998). Consequently, the box dimension may be assumed to be the corresponding index. Another significant fact is that the Horsehead is physically attached to its parent cloud so that the index may be assumed to apply to it as well, and so it may be inferred that the surrounding area is fractal as well and its fractal dimension is also 1.696572. This hypothesis is strengthened by an estimation of the box dimension of col. I (Fig. 4) in IC 434 (RA 5h 41m 48.89s; Dec $-2^{\circ} 17^{\prime} 52.57^{\prime \prime}$ ) to be 1.812614. Comparison with the box dimension of the Julia set by application of the Students t test shows that it is not significantly different. The presence of another head-trunk structure in Col. II (RA 5h 40m 55.89s; Dec $-2^{\circ} 17^{\prime} 52.57^{\prime \prime}$ ) in IC 434 also supports this hypothesis, although estimation of its box 
dimension has not been undertaken due to the presence of a bright star in front.

The assumption of a fractal morphology of Orion B can explain the clumpy or filamentary structure found. Kramer et al. (1996) have detected a total of 288 clumps in this region of which the 5 most massive are found near NGC 2024 and NGC 2023, both close to the Horsehead nebula. The clump masses are found to range from $14 M_{\odot}$ to $300 M_{\odot}$ and together they contain about $35 \%$ of the total clump mass. Physical association of the Orion B with Barnard's Loop (BL), the Orion association of OB stars, the Orion Reflection nebula, the Orion cloak, the Eridanus region and the extended shell of ionized and neutral hydrogen that contains BL and the Eridanus filaments (Goudis 1982; Thaddeus 1982), in turn, implies that the same fractal morphology may apply to these regions as well.

Growth of a dynamical system such as the Horsehead nebula and the region around it can be postulated to be described by the difference Eq. (8) under the following assumptions: every central point (repelling fixed point) is a seed (Witten \& Sander 1981; Meakin 1983; Maloy 1985); growth of cloud matter around such a central point occurs due to turbulence (Elmegreen 1999; Ossenkopf 1993) diffusion limited aggregation (DLA) (Vailati \& Giglio 1997; Botet et al. 1989) when turbulence has been significantly weakened and Brownian motion at higher densities (Ossenkopf 1993; Falconer 1997) giving rise to fractal structure and finally after the elapse of sufficient time, when adequate matter has accumulated around a central point, the formation of a dwarf, a star or a cluster of stars occurs, depending on the mass accrued. Such a model is successful in explaining spirals, filaments, bridges and the head-trunk structure found in Cols. I and II of IC 434 and the adjoining areas.

Acknowledgements. The author wishes to thank her guide, Prof. B. Basu, former Head of Department, Dept. of Applied Mathematics, University of Calcutta; Dr. N.K. Dey, Ramakrishna Mission Residential College, Narendrapur, Calcutta; S. Ray, Presidency College, Calcutta; the staff of the VBO, Kavalur especially M. Appakutty, G. Selvakumar, A. K. V. Rammana, E. Ramachandran, R. Sivakumar \& V. Ramesh for expert assistance during the observations and members of the Telescope committee, IIA, Bangalore, for observation time. Thanks also go to Prof. R. Gupta, Y. Wadedkar, Dr. Sridhar, Prof. J. V. Narlikar \& Dr. D. Mitra, IUCAA, Pune, C. D. Ravikumar, Cochin University,
India. Thanks also go to Dr. M. Hart \& Dr. Dixon, Department of Pure Mathematics, University of Sheffield and Prof. A. Boksenberg, Institute of Astronomy, Cambridge, UK.

\section{References}

Botet, R., Hegelsen, G., Skjeltrop, A. T., Mors, P. M., \& Jullien, R. 1989, Fractals Physical Origin \& Properties, ed. L. Piertonero (New York: Plenum Press), 259

Datta, S. 2001a, in Automated Data Analysis in Astronomy, ed. R. Gupta, H. P. Singh, \& C. Bailer-Jones (New Delhi: Narosa)

Datta, S. 2001b, Bull. Calcutta Math. Soc., 93, 451

Elmegreen, B. G. 1999, ApJ, 527, 266

Elmegreen, B. G., \& Falgarone, E. 1996, ApJ, 471, 816

Falconer, K. 1997, in Fractal Geometry (Chichester: Wiley \& Sons)

Frisch, U. 1999, Turbulence (CUP)

Goudis, C. 1982, in The Orion Complex: A Case Study of Interstellar Matter (Reidel)

Hetem, A., \& Lepine, J. R. D. 1993, A\&A, 270, 451

Kapur, J. N., \& Saxena, H. C. 1982, in Mathematical Statistics (New Delhi: S. Chand \& Co.)

Kramer, C., Stutzki, J., Ruihrig, R., \& Corneliussen, U. 1998, A\&A, 329,249

Kramer, C., Stutzki, J., \& Winnewisser 1996, A\&A, 307, 915

Lada, E. A., Bally, J., \& Stark, A. A. 1991, ApJ, 362, 432

Larson, R. B. 1981, MNRAS, 194, 809

Malin, D. F., Ogma, K., \& Walsh, J. R. 1987, MNRAS, 227, 361

Maloy, A. 1985, Phy. Rev. Lett., 85, 2688

Mandelbrot, B. B. 1982, in The Fractal Geometry of Nature (New York: Freeman)

Meakin, P. 1983, Phys. Rev. Lett., 51, 1119

Ossenkopf, V. 1993, A\&A, 280, 617

Pearson, E. S., \& Hartley, H. O. 1972, in Biometrica Tables for Statisticians, vol. II (CUP)

Peitgen, H. O., Jurgens, H., \& Saupe, D. 1992, in Introduction to Fractals and Chaos (New York: Springer-Verlag)

Seffens, W. 1999, Science, 285, 1228

Stutzki, J., Bensel, F., Heithausen, A., Ossenkopf, V., \& Zielinsky, M. 1998, A\&A, 336, 697

Vailati, A., \& Giglio, M. 1997, Nature, 390, 262

Thaddeus, P. 1982, Ann. New York Acad. Sci., 395, 9

Williams, J. P., Blitz, L., \& McKee, C. F. 2000, Protostars \& Planets IV, ed. V. Mannings, A. P. Boss \& S. S. Russell (Tucson: Univ. of Arizona Press), 97

Witten, T. A., \& Sanders, L. M. 1981, Phy. Rev. Lett., 47, 1400 